\title{
TNNT2 as a potential biomarker for the progression and prognosis of colorectal cancer
}

\author{
LI JING $^{1 *}$, LI FENG ${ }^{1 *}$, ZHIGUO ZHOU ${ }^{2}$, SHUAI SHI $^{3}$, RUOYING DENG $^{3}$, \\ ZHICONG WANG $^{3}$, YI ZHANG ${ }^{3}$, ZHIXUE REN ${ }^{4}$ and YIBING LIU ${ }^{1}$ \\ Departments of ${ }^{1}$ Medical Oncology and ${ }^{2}$ Radiotherapy, Fourth Hospital of Hebei Medical University; \\ ${ }^{3}$ Hebei Medical University, Shijiazhuang, Hebei 050011; ${ }^{4}$ The Seven People's Hospital of Hebei Province, \\ Dingzhou, Hebei 073000 , P.R. China
}

Received September 30, 2019; Accepted February 26, 2020

DOI: $10.3892 /$ or.2020.7637

\begin{abstract}
Colorectal cancer (CRC) is the third most common cancer worldwide. At present, there are limited effective biomarkers of CRC. The present study aimed to identify potential signatures associated with the tumorigenesis and prognosis of CRC using publicly available databases, and further validate the identified biomarkers in CRC cell lines. Identification of differentially expressed mRNAs between CRC and paracancerous samples was conducted based on data from The Cancer Genome Atlas (TCGA; 471 tumor samples and 41 normal samples). Survival analysis was performed to explore the prognostic value of troponin 2 (TNNT2) in the TCGA training set, which was further validated in an external dataset, GSE17531. Functional enrichment analysis was conducted to determine the possible biological functions using GSEA 3.0. Reverse transcription-quantitative PCR (RT-qPCR) and western blotting were utilized to detect the mRNA and protein expression levels of TNNT2 between CRC and normal colorectal cells. Immunohistochemistry was performed to detect the protein expression of TNNT2 in CRC and normal tissues. TNNT2 was significantly upregulated in CRC samples compared with adjacent normal samples in the TCGA dataset. Increased expression of TNNT2 was associated with inferior prognosis in the TCGA training dataset and GSE17531 validation dataset. Functional enrichment analysis revealed that the ErbB signaling pathway and glycerophospholipid metabolism pathway were significantly activated in the TNNT2 high expression group. Overexpression of TNNT2 mRNA and TNNT2 protein in CRC tumor cells was confirmed by RT-qPCR and western blotting, respectively.
\end{abstract}

Correspondence to: Dr Yibing Liu, Department of Medical Oncology, Fourth Hospital of Hebei Medical University, 12 JianKang Road, Shijiazhuang, Hebei 050011, P.R. China

E-mail: liuyibingsjz@outlook.com

${ }^{*}$ Contributed equally

Key words: colorectal cancer, troponin 2 gene, tumorigenesis, prognosis
Immunohistochemistry indicated increased protein expression levels of TNNT2 in CRC tissues in comparison with normal tissues. TNNT2 was associated with the tumorigenesis and prognosis of CRC, which may be useful for novel biomarker identification and targeted therapeutic strategy development.

\section{Introduction}

Colorectal cancer (CRC) is the third most common cancer and the second leading cause of cancer-associated mortalities worldwide (1). The incidence rate of CRC in China has been nearly three-fold lower than that in Europe $(2,3)$; however, its incidence has increased rapidly in recent years $(4,5)$. CRC is more frequent in patients ranged 40-70 years old; however, an increasing number of patients are diagnosed with $\mathrm{CRC}$ under 40 years of age (6). Currently, the underlying mechanism of CRC carcinogenesis remains unclear. Due to improvement in early detection and surgical treatment, the prognosis of patients with CRC with localized tumors has been significantly enhanced (7). However, the prognosis for patients with advanced tumors remains poor (8). Moreover, although clinical staging is useful to some extent to predict CRC prognosis, patient outcomes can vary significantly even for patients with similar clinical features (9). Heterogeneity in somatic or germline changes may substantially affect patient prognosis.

Altered mRNA expression of genes involved in tumor proliferation, autophagy and transcription has been reported to play an important role in predicting the onset or survival outcome of CRC (10-12). However, the number of studies investigating mRNAs in order to predict the onset and prognosis of CRC remains limited. A previous study reported that cyclooxygenase-2 mRNA upregulation was associated with CRC carcinogenesis using reverse transcription-quantitative PCR (RT-qPCR) based on 60 CRC tumor tissues and normal tissues (13). Another study reported that increased expression of rabaptin-5-associated exchange factor mRNA was associated with poor survival of CRC using RT-qPCR and survival analysis based on $187 \mathrm{CRC}$ tumor tissues (14). Wang et al (15) established a 31-gene expression classifier to predict CRC recurrence using a gene expression microarray based on $281 \mathrm{CRC}$ samples. However, these studies evaluated only a single clinical outcome (progression or prognosis), and lacked laboratory-based 
validation experiments, restricting the possible application of these reported mRNAs in clinical practices. Therefore, it is imperative to identify and validate key mRNAs associated with the carcinogenesis and prognosis of CRC in order to further facilitate the development of new targeted therapies.

Substantial developments in high-throughput transcriptome sequencing and microarray technologies have provided opportunities to identify novel mRNA biomarkers associated with the tumorigenesis and prognosis of CRC. In the present study, differential expression analysis was performed to explore critical genes in CRC. Survival analysis was performed to evaluate the prognostic value of TNNT2. Functional enrichment analysis was employed to explore the potential biological processes involved in CRC tumorigenesis and prognosis. Epidermal growth factor receptor (EGFR) is a key factor for the ErbB signaling pathway involved in tumor growth $(16,17)$. In addition, Fatty acid synthase (Fasn) is the key metabolic enzyme that accounts for the glycerophospholipid metabolism pathway to regulate the proliferation and migration in various tumors $(18,19)$. Therefore, EGFR and Fasn were selected as a biomarker for the two respective signaling pathways. RT-qPCR and western blotting were conducted to measure the mRNA and protein expression levels of TNNT2 between CRC tumor and normal cells. The protein expression of TNNT2 in CRC and normal tissues was detected via immunohistochemistry.

In summary, the present study investigated the expression of TNNT2 at the mRNA and protein level in CRC and clarified the correlation between the TNNT2 expression and clinicopathological parameters.

\section{Materials and methods}

Data sources. The data of CRC tumor and adjacent normal tissue samples were obtained from The Cancer Genome Atlas (TCGA; www.cancergenome.nih.gov) and the Gene Expression Omnibus (GEO; https://www.ncbi.nlm.nih. gov/geo/; Access number: GSE17537) databases. The TCGA-CRC dataset contained a total of 512 CRC samples, including 471 tumor samples and 41 adjacent normal samples. The age of the samples is from 41 to 90 with the median age of 68. Numbers of female and male patients is 212 and 235, respectively. The GEO dataset contained 55 CRC tumor samples. The age of those 55 patients is from 23 to 94 with the median age of 61 . Numbers of female and male patients is 29 and 26 , respectively.

Differential expression analysis. Differential expression analysis was performed on the TCGA-CRC dataset using edgeR package in R v. 3.5.3 software (20). First, genes were excluded with average counts $<10$. Then the samples were divided into normal tissue group ( $\mathrm{N}$ ) and tumor tissue group $(\mathrm{T})$ according to the sample type. A false discovery rate (FDR) $<0.05$ and $\mid \log 2$ fold change $(\mathrm{FC}) \mid>1$ were set as the criteria for screening differentially expressed genes.

Gene set enrichment analysis (GSEA). GSEA (version 2.2) was conducted for functional enrichment analysis (21). The selected gene set was Kyoto Encyclopedia of Genes and Genomes (KEGG) pathway. $\mathrm{P}<0.05$ was set as the threshold for screening significantly enriched KEGG pathways.
Table I. Primer sequences for reverse transcription-quantitative PCR.

\begin{tabular}{ll}
\hline Gene & \multicolumn{1}{c}{ Primer sequence $\left(5^{\prime} \rightarrow 3^{\prime}\right)$} \\
\hline TNNT2 & F: TTCACCAAAGATCTGCTCCTCGCT \\
& R:TTATTACTGGTGTGGAGTGGGTGTGG \\
EGFR & F: TCCCTCAGCCACCCATATGTAC \\
& R: GTCTCGGGCCATTTTGGAGAATTC \\
Fasn & F: ATGCGGGACAGAGCAACTACGG \\
& R: CAGCCTTCTCAGCCAGCACAAA \\
GAPDH & F: GGTGAAGGTCGGTGTGAACG \\
& R: CTCGCTCCTGGAAGATGGTG
\end{tabular}

F, forward; R, reverse; TNNT2, troponin T2.

Cell culture. The normal colorectal cell line FHC, colon cancer cell line LoVo, CRC cell line SW620, and colon cancer cell line SW1116 were purchased from BeNa Culture Collection. FHC, LoVo, SW620 and SW1116 cells were cultured in DMEM (Gibco; Thermo Fisher Scientific, Inc.), DMEM/F12 (Gibco; Thermo Fisher Scientific, Inc.), DMEM/F-12K (Gibco; Thermo Fisher Scientific, Inc.) and DMEM/L-15 (Gibco; Thermo Fisher Scientific, Inc.) containing $10 \%$ fetal bovine serum (FBS; Hyclone; GE Healthcare Life Sciences) and $1 \%$ penicillin/streptomycin (Hyclone; GE Healthcare Life Sciences), respectively. The cells were incubated at $37^{\circ} \mathrm{C}$ with $5 \% \mathrm{CO}_{2}$.

Overexpression of TNNT2. RNA was extracted from LoVo cells using Trizol reagent (Thermo Fisher Scientific, Inc.). cDNA was synthesized by TransScript ${ }^{\circledR}$ Two-Step RT-PCR SuperMix (TransGen Biotech). ORF sequence of TNNT2 was amplified via RT-PCR according to the manufacturer's instructions of $2 x_{\text {TransTaq }}^{\circledR}$ HiFi PCR SuperMix II (TransGen Biotech). TNNT2 primer sequences were as follows: Forward, 5'-ATGTCTGACATAGAAGAGGTGGTGG-3' and reverse, 5'-CTATTTCCAGCGCCCGGTGACTTTA-3'. PCR reaction condition was as follows: $94^{\circ} \mathrm{C} 3 \mathrm{~min}, 1$ cycle, $94^{\circ} \mathrm{C} 30 \mathrm{sec}$, $55^{\circ} \mathrm{C} 30 \mathrm{sec}, 72^{\circ} \mathrm{C} 3 \mathrm{~min}, 35$ cycles and $72^{\circ} \mathrm{C} 10 \mathrm{~min}, 1$ cycle. Then PCR products were inserted into the pCMV-Myc vector (Biovector Co., Ltd.) to construct the expression vector pCMV-Myc-TNNT2. LoVo, SW620 and SW1116 cells were transfected with $16 \mu \mathrm{g} / \mathrm{ml}$ pCMV-Myc-TNNT2 using Lipofectamine 2000 (Thermo Fisher Scientific, Inc.) for $48 \mathrm{~h}$ according to the manufacturer's instructions.

$R T-q P C R$. Total RNA of FHC, LoVo, SW620 and SW1116 cells was extracted using Trizol (Invitrogen; Thermo Fisher Scientific, Inc.). The purity and concentration of RNA were determined via NanoDrop ND-2000 spectrophotometer (NanoDrop Technologies; Thermo Fisher Scientific, Inc.). Then the RNA was reverse transcribed into cDNA using TransScript ${ }^{\circledR}$ Two-Step RT-PCR SuperMix (TransGen Biotech) according to the manufacturer's instructions. RT-qPCR was performed according to the manufacturer's instructions of the SYBR Premix Ex Taq kit (Roche Diagnostics), with GAPDH used as the internal reference. The thermocycling conditions 

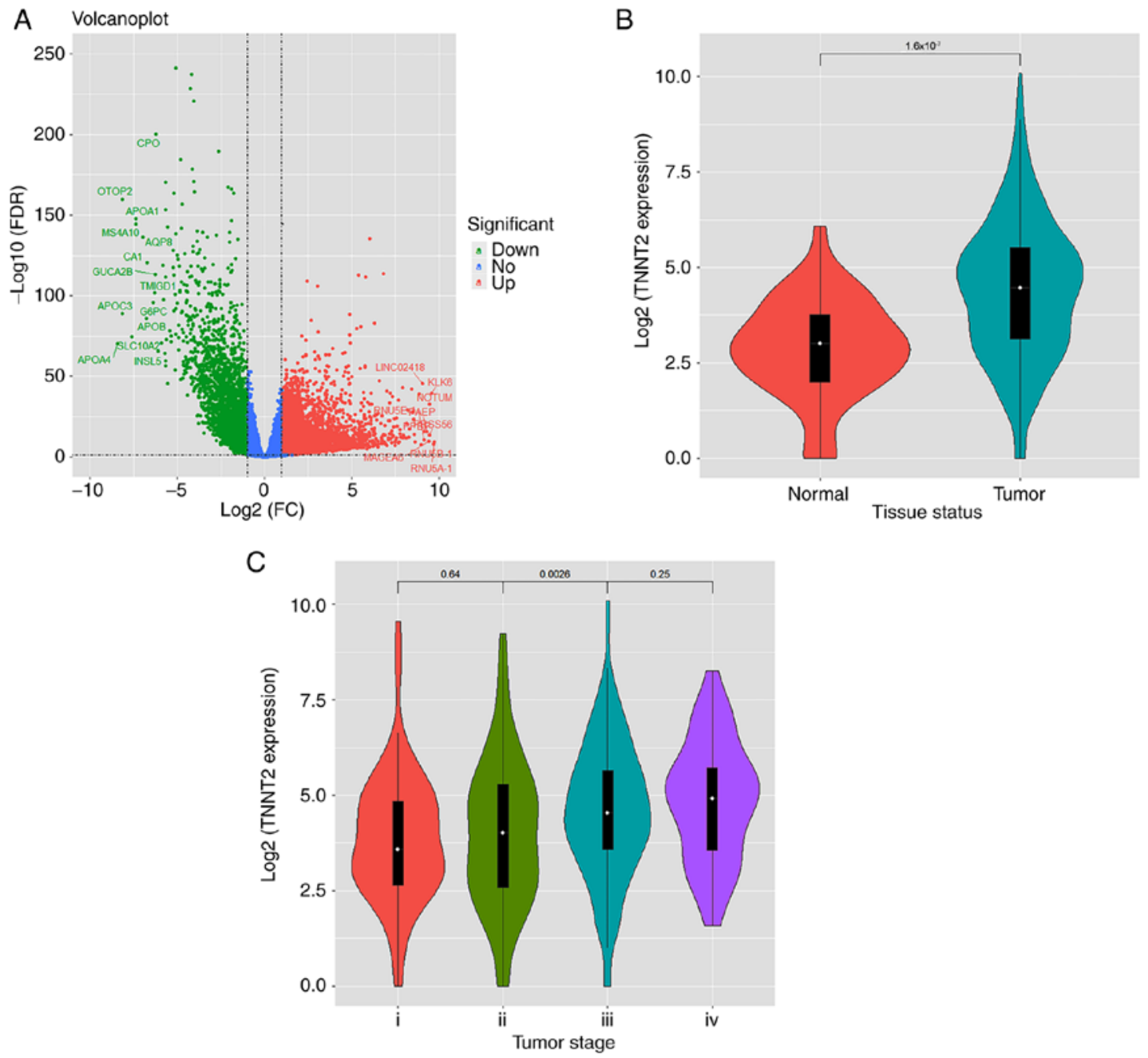

Figure 1. Elevated TNNT2 expression is associated with the tumorigenesis and progression of CRC. (A) Volcano plot of differentially expressed genes between $\mathrm{CRC}$ and paracancerous tissues. Green plots represented significantly downregulated genes in CRC tumor tissues. Red plots represented significantly upregulated genes in CRC tumor tissues. Blue plots represented genes that were not significantly differentially expressed between CRC tumor and normal tissues. (B) Box plot of TNNT2 mRNA expression levels between CRC tumor and normal tissues. (C) Box plot of TNNT2 mRNA expression levels among different pathological stages of CRC. TNNT2, troponin T2; CRC, colorectal cancer.

were as follows: Step $1,95^{\circ} \mathrm{C}$ for $5 \mathrm{~min}$; step $2,95^{\circ} \mathrm{C}$ for $30 \mathrm{sec}$; step $3,58-65^{\circ} \mathrm{C}$ for $30 \mathrm{sec}$; step $4,72^{\circ} \mathrm{C}$ for $30 \mathrm{sec}$; step 5, repeat from step 2 for 34 cycles; step $6,72^{\circ} \mathrm{C}$ for $10 \mathrm{~min}$. The relative expression of TNNT2 was calculated using the $2^{-\Delta \Delta C q}$ method. The primer sequences of RT-qPCR are listed in Table I.

Western blotting. The cells were lysed using RIPA solution and then total proteins were extracted. The proteins were quantified using bovine serum albumin (BSA; Boster Biological Technology Co., Ltd). The protein samples were electrophoresed on 10\% SDS-PAGE and transferred onto PVDF membranes. After blocking with 1X Blotto (Thermo Fisher Scientific, Inc.) for $2 \mathrm{~h}$ at room temperature, the membranes were incubated with anti-Cardiac Troponin $\mathrm{T}$ antibody (cat. no. ab91605; 1:1,000; Abcam) and anti-GAPDH antibody (cat. no. ab8245; 1:1,000; Abcam) at $4^{\circ} \mathrm{C}$ overnight. After washing with TBST three times at room temperature, the membranes were incubated with horseradish peroxidase (HRP)-labeled goat anti-rabbit IgG as the secondary antibody (cat. no. ab6728; 1:1,000; Abcam) for $1.5 \mathrm{~h}$ at room temperature. The proteins were visualized using Western Lightning ${ }^{\text {TM }}$
Chemiluminescence reagent (PerkinElmer, Inc.), and densitometric analysis was conducted using LabWorks ${ }^{\mathrm{TM}} 4.6$ (UVP, LLC). GAPDH was used as the internal control.

Immunohistochemistry. The $\mathrm{CRC}$ and normal tissues were fixed using $4 \%$ paraformaldehyde at $4{ }^{\circ} \mathrm{C}$ overnight, embedded with paraffin, and sliced into 3-5 $\mu \mathrm{m}$ sections, followed by dewaxing. After antigen retrieval by microwave, the sections were blocked with 10\% normal goat serum (Beijing Solarbio Science \& Technology Co., Ltd.) and incubated at room temperature for $20 \mathrm{~min}$. Then the primary antibody rabbit anti-cardiac troponin T (cat. no. ab91605; Abcam) were added for an incubation at $4^{\circ} \mathrm{C}$ overnight. The biotin-labeled $\operatorname{IgG}$ (cat. no. Sp-9001; 1: 1,000; OriGene Technologies, Inc.) was used as secondary antibody for $30 \mathrm{~min}$ at room temperature. After development in DAB and counterstaining with hematoxylin for $5 \mathrm{~min}$ at room temperature, the sections were sealed with resinene. Five fields were randomly selected and imaged under an optical microscope (BX51T-PHD-J11, Olympus Corporation). The images were analyzed using Image-Pro Plus (Media Cybernetics, Inc.). 

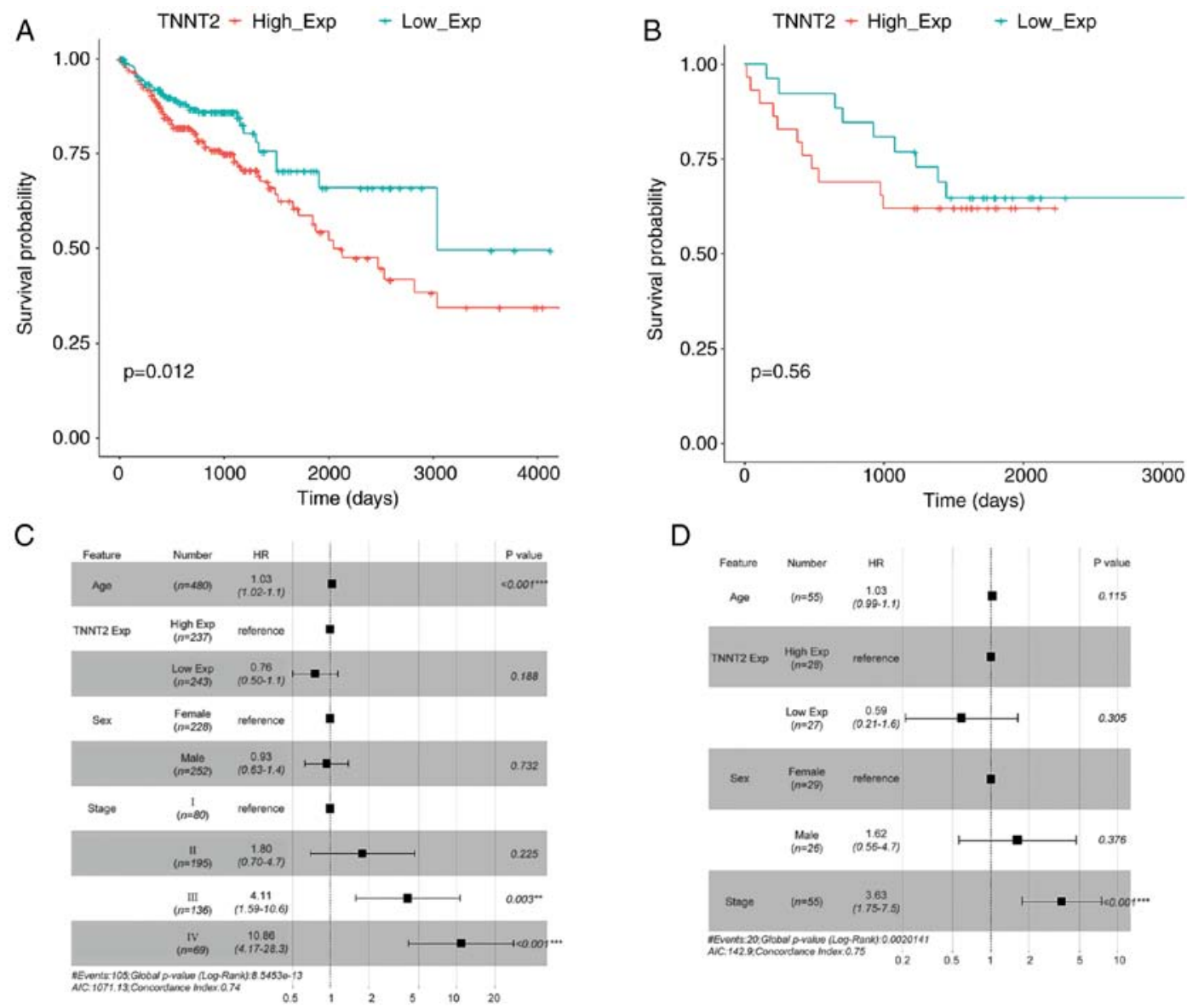

Figure 2. Elevated TNNT2 expression is associated with poor prognosis in patients with CRC. (A) Kaplan-Meier survival curves for CRC patients between high- and low-TNNT2 expression groups in TCGA. The blue curve represents the low expression group. The red curve represents the high expression group. (B) Kaplan-Meier survival curves for patients with CRC between high- and low-TNNT2 expression groups in GSE17537. The blue curve represents the low-TNNT2 expression group. The red curve represented high-TNNT2 expression group. (C) Cox regression analysis adjusting for age, sex, staging and $T N N T 2$ expression level (high or low) in TCGA. P $<0.1$ was set as the cutoff criteria for screening genes significantly associated with CRC survival. (D) Cox regression analysis adjusting for age, sex, staging and TNNT2 expression level (high or low) in GSE17537. $\mathrm{P}<0.1$ was set as the cutoff criteria for screening genes significantly associated with CRC survival. TNNT2, troponin T2; CRC, colorectal cancer.

Statistical analysis. Cox regression analysis was performed by using survival and survminer packages in R software (22). Clinical factors, including age, sex, TNM staging system [American Joint Committee on Cancer (AJCC) (23) and the International Union Against Cancer (UICC) (24)] and gene expression levels, were adjusted in the Cox regression model. $\mathrm{P}<0.1$ was set as the threshold to screen factors significantly associated with CRC survival. Kaplan-Meier analysis was performed by stratifying CRC patients according to median $T N N T 2$ expression. Data were presented as the mean \pm SEM, $\mathrm{n}=3$. The significance was assessed via one-way ANOVA (with Tukey's post hoc analysis). Statistical analysis was performed using GraphPad Prism Version 5.0 software (GraphPad Software, Inc.).

\section{Results}

TNNT2 is upregulated in CRC samples compared with paracancerous samples. A total of 2,703 downregulated genes and 4,001 upregulated genes between CRC tumor samples and paracancerous samples were identified. The volcano blot is presented in Fig. 1A. Analysis of these differentially expressed genes revealed that $T N N T 2$ was significantly upregulated in
CRC tumor samples vs. adjacent normal samples, as indicated by Fig. 1B $\left(\mathrm{P}=1.6 \times 10^{-7}\right)$. Furthermore, there was a positive association between TNNT2 gene expression with the stages of patients with CRC, especially between stage II and stage III, as indicated in Fig. $1 \mathrm{C}(\mathrm{P}=0.0026)$. As the association between TNNT2 and CRC has not been investigated in previous studies, TNNT2 was selected as the key candidate gene, and the association of TNNT2 with the progression and prognosis of CRC was investigated.

Elevated TNNT2 is associated with poor prognosis in CRC. CRC samples were divided into a high- and low-expression group according to the median level of TNNT2 expression which was 4.82 (log2-based) according to the survival package. The survival curve was plotted using survminer package (Fig. 2A). It was revealed that the expression level of TNNT2 had a significant effect on survival outcome $(\mathrm{P}=0.012)$. Moreover, a Cox regression model was constructed adjusting for age, sex, staging and TNNT2 expression level (high or low) (Fig. 2C). It was revealed that compared with the high-TNNT2 expression group, the low-expression group exhibited a more favorable survival outcome. Additionally, patients with CRC with higher stages had a higher risk of mortality. 


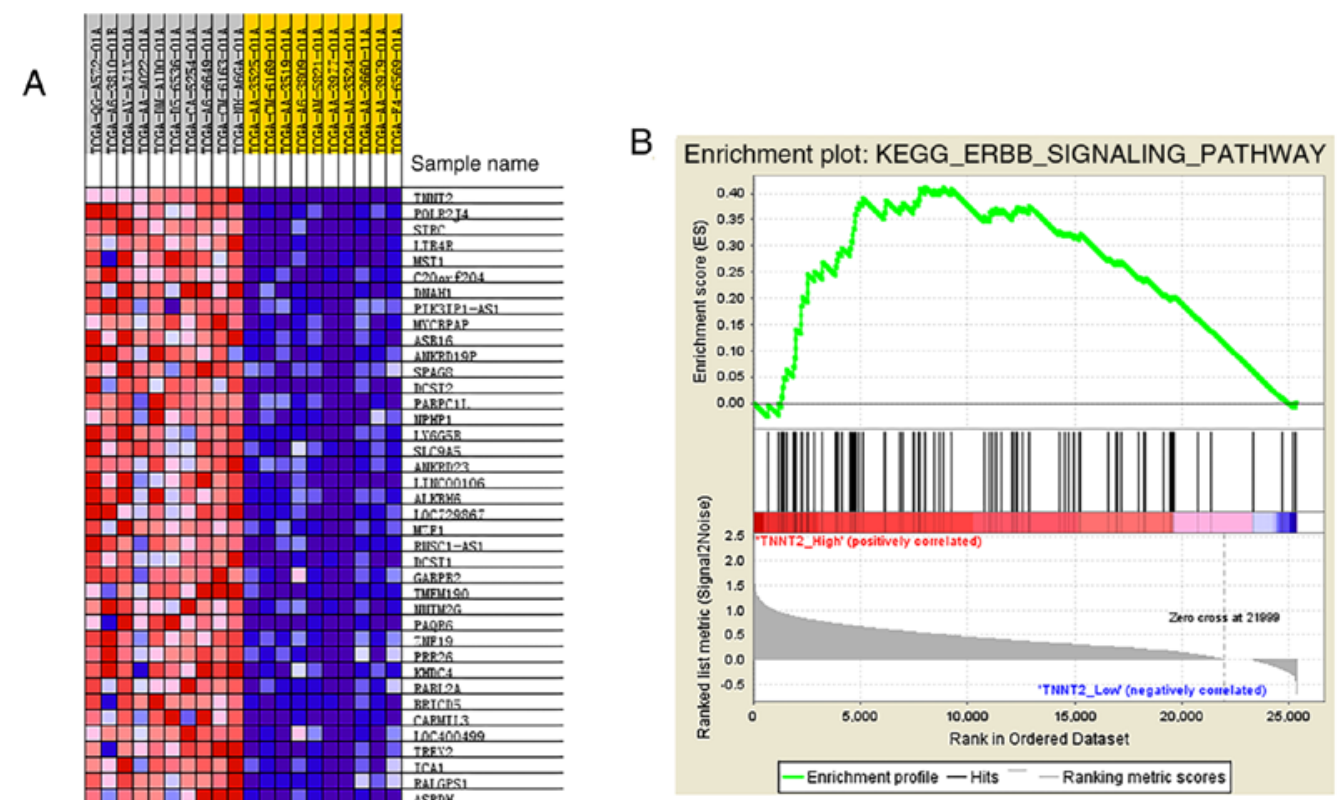

C

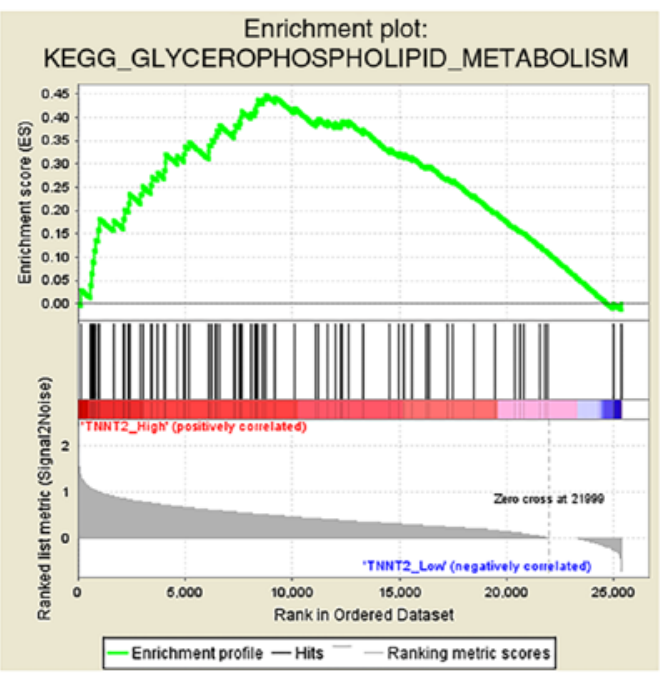

Figure 3. Increased TNNT2 expression may influence the tumorigenesis and prognosis of CRC via activation of ErbB and glycerophospholipid metabolism pathways. (A) Heatmap of the selected 200 genes according to tbe $20 \mathrm{CRC}$ samples with the top 10 and the bottom 10 TNNT2 expression levels among all CRC samples. (B) The ErbB signaling pathway was significantly activated in CRC samples with high TNNT2 expression. (C) The glycerophospholipid metabolism pathway was significantly activated in CRC samples with high TNNT2 expression. TNNT2, troponin T2; CRC, colorectal cancer.

Validation of the prognostic effect of TNNT2 in a GEO cohort. The same method was utilized for the survival analysis to verify the association of TNNT2 expression with survival in GEO dataset GSE17537. The survival curve suggested that different expression levels of TNNT2 had an effect on survival in patients with CRC, but the difference was not statistically significant $(\mathrm{P}=0.56)$. The Cox regression analysis indicated that the TNNT2 low expression group had a lower risk of mortality compared with the high expression group, and patients at higher stages had a higher risk of mortality (Fig. 2B and D).
Significantly enriched pathways in CRC samples with high TNNT2 expression. According to the median expression level of TNNT2, CRC samples were stratified into high and low expression groups, and then differential expression analysis was performed. A total of 784 differentially expressed genes were identified, of which 163 genes were downregulated and 621 genes were upregulated. The top 100 differentially expressed genes within down- and upregulated gene sets were selected, respectively. A heatmap was constructed comprising the 200 selected genes in $10 \mathrm{CRC}$ samples with the highest and lowest TNNT2 expression, respectively (Fig. 3A). According to the 
A

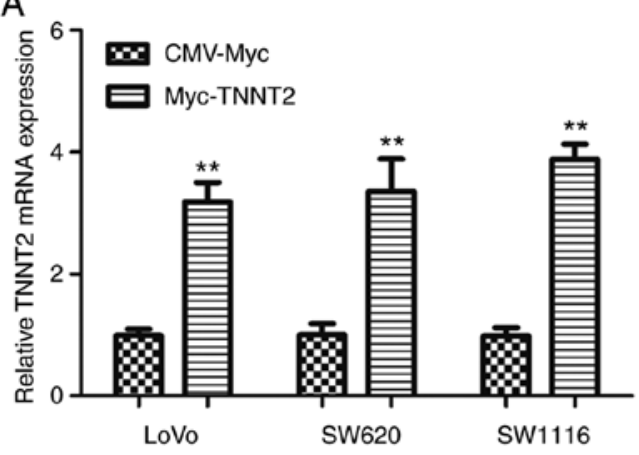

B

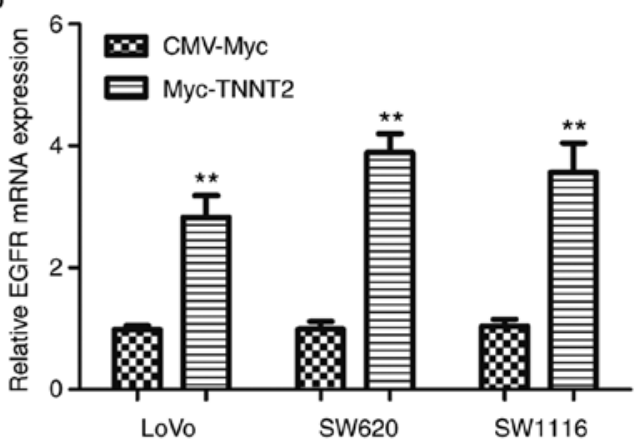

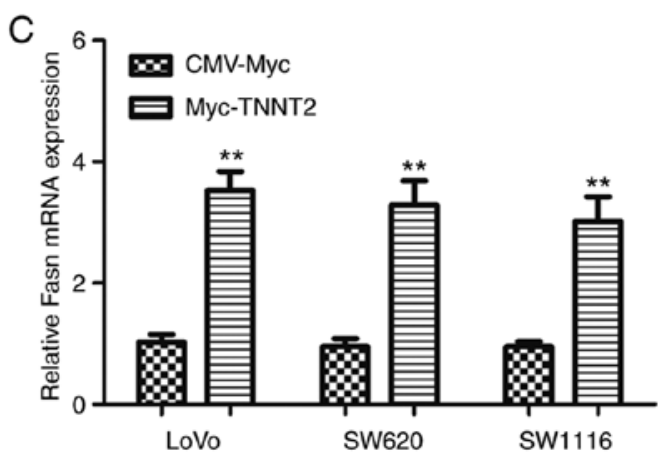

Figure 4. TNNT2 overexpression upregulates the ErbB signaling pathway and glycerophospholipid metabolism pathway in CRC cell lines. (A) Overexpression of TNNT2 in LoVo, SW620 and SW1116 cells transfected with pCMV-Myc-TNNT2 or empty vector, then the expression of TNNT2 was validated by RT-PCR. (B) TNNT2 overexpression upregulated EGFR expression. (C) TNNT2 overexpression upregulated Fasn expression. ${ }^{* *} \mathrm{P}<0.01$ vs. the control group. TNNT2, troponin T2; CRC, colorectal cancer; EGFR, epidermal growth factor receptor; Fasn, fatty acid synthase.

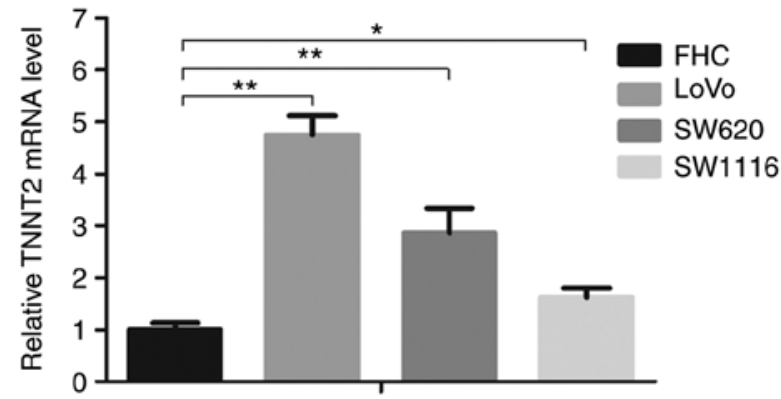

Figure 5.TNNT2 mRNA expression levels among normal colorectal cell line (FHC) and CRC cell lines (LoVo, SW620 and SW1116). Data were reported as mean \pm SEM. Significance was assessed by one-way analysis of ANOVA and Tukey's post hoc analysis. ${ }^{*} \mathrm{P}<0.05 ;{ }^{* *} \mathrm{P}<0.01$ vs. the control group. TNNT2, troponin T2; CRC, colorectal cancer.

functional enrichment analysis, the 'ErbB signaling pathway' and 'glycerophospholipid metabolism pathway' were significantly activated in the high TNNT2 expression group (Fig. 3B).

TNNT2 overexpression upregulates the ErbB signaling and glycerophospholipid metabolism pathways in CRC cell lines. In order to further confirm the effect of TNNT2 on the ErbB signaling pathway and glycerophospholipid metabolism pathway, RT-PCR were carried out in CRC cell lines transfected with the expression vector pCMV-Myc-TNNT2. As indicated in Fig. 4A, TNNT2 expression was significantly increased in LoVo, SW620 and SW1116 cells following transfection with pCMV-Myc-TNNT2. Additionally, the expression of EGFR and Fasn were significantly increased (Fig. 4B and C). This
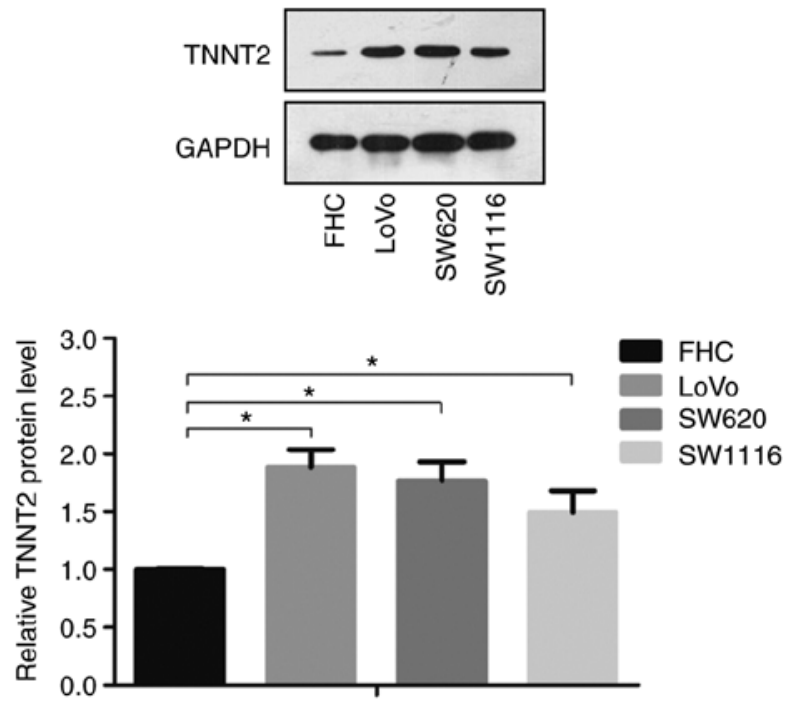

Figure 6. TNNT2 protein expression levels between a normal colorectal cell line (FHC) and CRC cell lines (LoVo, SW620 and SW1116). The data were reported as mean \pm SEM. Significance was assessed via one-way ANOVA and Tukey's post hoc analysis. ${ }^{*} \mathrm{P}<0.05$ vs. the control group. TNNT2, troponin $\mathrm{T} 2$; CRC, colorectal cancer.

result demonstrated that high TNNT2 expression upregulates the ErbB signaling and glycerophospholipid metabolism pathway in CRC cell lines, which was consistent with the present bioinformatics analysis.

Validation of elevated TNNT2 expression in CRC cell lines and tissues. The mRNA and protein expression levels of 
A
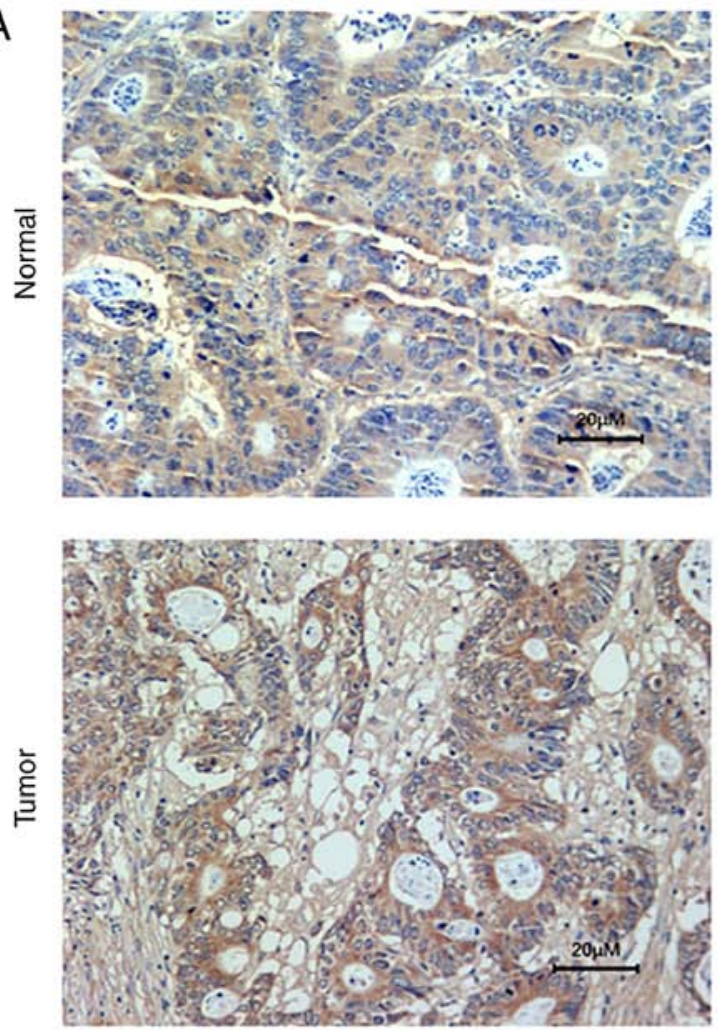

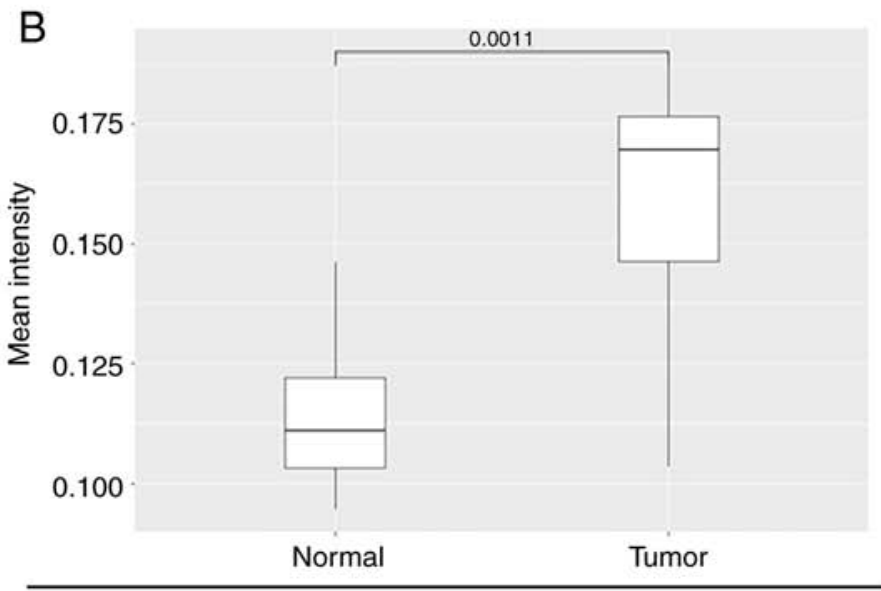

\begin{tabular}{ccc}
\hline No. & 10 & 10 \\
\hline Mean & 0.111 & 0.170 \\
\hline
\end{tabular}

Figure 7. Protein expression levels of TNNT2 in CRC tissues are significantly higher compared with normal tissues. (A) Immunohistochemical staining of CRC and normal tissues. (B) Mean intensity of CRC and normal tissues $(\mathrm{P}<0.05)$. TNNT2, troponin T2; CRC, colorectal cancer.

TNNT2 were detected in normal colorectal cell line FHC, colon cancer cell line LoVo, CRC cell line SW620 and colon cancer cell line SW1116. The result revealed that compared with the normal colorectal cells, the mRNA expression level of TNNT2 was significantly increased in the CRC cells $(\mathrm{P}<0.05$; Fig. 5). Moreover, the protein expression level of TNNT2 in CRC cells was higher compared with in normal colorectal cells $(\mathrm{P}<0.05$; Fig. 6). Immunohistochemistry was performed to detect the protein expression of TNNT2 in CRC and normal tissues. The result indicated increased protein expression level of TNNT2 in CRC tissues in comparison to that in normal tissues $(\mathrm{P}<0.05$; Fig. 7$)$.

\section{Discussion}

In the present study, the potential mRNA signatures associated with CRC risk were explored using datasets from publicly available databases. Differential expression analysis in TCGA cohort indicated that TNNT2 was significantly upregulated in CRC samples and may be associated with the onset of CRC. Survival analysis was performed to investigate the potential of TNNT2 as a prognostic biomarker. The carcinogenic and prognostic value of TNNT2 was further replicated in an external validation dataset, GSE17537. Furthermore, laboratory work was also conducted to confirm the increased mRNA and protein expressions of TNNT2 in CRC cell lines. The RT-qPCR and western blot analyses revealed that TNNT2 mRNA and protein expressions were significantly elevated in CRC cell lines compared with normal colorectal cell lines, which further validated the present analysis.
TNNT2 encodes the tropomyosin-binding subunit of the troponin complex, which controls muscle contraction in response to differential concentrations of intracellular calcium ions $(25,26)$. Several studies have reported the association between abnormal TNNT2 mRNA splicing and dilated cardiomyopathy risk $(27,28)$. Typically, altered mRNA splicing generates different transcripts, which are then translated into protein isoforms and influence diverse biological processes (29). However, abnormal mRNA splicing may result in significant changes to protein structure and function, thus resulting in tumorigenesis (30). Lokody and Isabel (31) reported that aberrant mRNA splicing promotes the growth of colon tumors via upregulation of PRPF6, a driver of colon carcinogenesis. According to genome-wide profiling of alternative splicing events, Zong et al (32) identified 13 genes with differential mRNA splicing patterns, which may predict colorectal cancer prognosis. The present study demonstrated that TNNT2 was differentially expressed between CRC tumor and adjacent normal tissues, based on both bioinformatics analysis and experimental strategies. Normal colon tissue is composed of smooth muscle. TNNT2 is expressed at a low level in normal colon tissue, which is consistent with the present results (33). Therefore, it was speculated that TNNT2 may not serve a physiological role in normal colon tissue. A significant difference in TNNT2 mRNA expression between different tumor stages was also identified. Thus, it was hypothesized that the altered mRNA and protein expression levels of TNNT2 in CRC cells may be induced by abnormal TNNT2 mRNA splicing, which may result in colorectal carcinogenesis. 
According to the current functional enrichment analysis, the 'ErbB signaling pathway' was significantly activated in the high TNNT2 expression group. Upregulation, or mutation of several members of the ErbB pathway including EGFR, ErbB2 and ErbB3, have been identified in numerous cancer types (34). By modulating extracellular matrix components, ErbB recepters serve a notable role in tumor proliferation and metastasis (35). Upregulation of EGFR, a member of the ErbB family, is associated with the onset and prognosis of colorectal cancer, via activating multiple pathways, such as the MAPK and PI3K pathways (36). The current finding that differentially expressed genes in the high TNNT2 expression group were enriched in the ErbB signaling pathway was in accordance with previous studies and suggested that elevated TNNT2 expression might influence CRC tumorigenesis and prognosis through regulating the ErbB signaling pathway. In addition, the current study identified that the glycerophospholipid metabolism pathway was also activated in CRC samples with high TNNT2 expression. Based on global lipid omics analysis, Hung et al (37) reported that diverse glycerophospholipid levels were associated with chromosome instability in gastric cancer. Several studies have reported an association between alteration of lipid metabolism and the risk of colorectal cancer $(38,39)$. The results of the present study were consistent with previous studies and suggested that high expression of TNNT2 may affect CRC tumorigenesis via regulation of glycerophospholipid metabolism.

In conclusion, the present study revealed that elevated TNNT2 expression is associated with the tumorigenesis and prognosis of CRC, which may facilitate the identification of novel biomarkers and the development of targeted therapeutic strategies.

\section{Acknowledgements}

Not applicable.

\section{Funding}

No funding was received.

\section{Availability of data and materials}

The datasets used and/or analyzed during the present study are available from the corresponding author on reasonable request.

\section{Authors' contributions}

LJ and LF contributed to the design of the study, wrote the manuscript and analyzed the data. ZZ, SS and RD revised the manuscript and contributed to the design of the study. ZW, YZ and ZR acquired, analyzed and interpreted the data. YL made substantial contributions to the conception and design of the present study and revised the manuscript. All authors read and approved the final manuscript.

\section{Ethics approval and consent to participate}

Written informed consent was obtained from all participants and the present study was approved by the Ethics Committee of Fourth Hospital of Hebei Medical University (Shijiazhuang, China; approval no. 2019MEC107).

\section{Patient consent for publication}

Not applicable.

\section{Competing interests}

The authors declare that they have no competing interests.

\section{References}

1. Bray F, Ferlay J, Soerjomataram I, Siegel RL, Torre LA and Jemal A: Global cancer statistics 2018: GLOBOCAN estimates of incidence and mortality worldwide for 36 cancers in 185 countries. CA Cancer J Clin 68: 394-424, 2018.

2. Douaiher J, Ravipati A, Grams B, Chowdhury S, Alatise O and Are C: Colorectal cancer-global burden, trends, and geographical variations. J Surg Oncol 115: 619-630, 2017.

3. Favoriti P, Carbone G, Greco M, Pirozzi F, Pirozzi RE and Corcione F: Worldwide burden of colorectal cancer: A review. Updates Surg 68: 7-11, 2016.

4. Chen W, Zheng R, Baade PD, Zhang S, Zeng H, Bray F, Jemal A, Yu XQ and He J: Cancer statistics in China, 2015. CA Cancer J Clin 66: 115-132, 2016.

5. Zheng R, Zeng H, Zhang S, Chen $\mathrm{T}$ and Chen W: National estimates of cancer prevalence in China, 2011. Cancer Lett 370: 33-38, 2016.

6. Karanikas $M$ and Esebidis A: Increasing incidence of colon cancer in patients $<50$ years old: A new entity? Ann Transl Med 4: 164, 2016.

7. Zhang X, Shao S, Gao Y, Zhang M and Lu Y: Meta-analysis of relationship between extranodal tumor deposits and prognosis in patients with colorectal cancer. Zhonghua Wei Chang Wai Ke Za Zhi 19: 334-338, 2016 (In Chinese).

8. Brenner H, Kloor M and Pox CP: Colorectal cancer. Lancet 383: 1490-1502, 2014

9. Yusup A, Wang HJ, Rahmutula A, Sayim P, Zhao ZL and Zhang GQ: Clinical features and prognosis in colorectal cancer patients with different ethnicities in Northwest China. World J Gastroenterol 41: 7183-7188, 2013.

10. Ren Q and Jin B: The clinical value and biological function of PTTG1 in colorectal cancer. Biomed Pharmacother 89: 108-115, 2017.

11. Gil J, Ramsey D, Szmida E, Leszczynski P, Pawlowski P, Bebenek $M$ and Sasiadek MM: TheBAXgene as a candidate for negative autophagy-related genes regulator on mRNA levels in colorectal cancer. Med Oncol 34: 16, 2017.

12. Mansour MA and Senga T: HOXD8 exerts a tumor-suppressing role in colorectal cancer as an apoptotic inducer. Int J Biochem Cell Biol 88: 1-13, 2017.

13. Roelofs HM, Te Morsche RH, van Heumen BW, Nagengast FM and Peters WH: Over-expression of COX-2 mRNA in colorectal cancer. BMC Gastroenterology 14: 1, 2014.

14. Zhang JS, Yang LQ, Du BR and Gao H: Higher RABEX-5 mRNA predicts unfavourable survival in patients with colorectal cancer. Eur Rev Med Pharmacol Sci 21: 2372-2376, 2017.

15. Wang L, Shen X, Wang Z, Xiao X, Wei P, Wang Q, Ren F, Wang Y, Liu Z, Sheng W, et al: A molecular signature for the prediction of recurrence in colorectal cancer. Mol Cancer 14: 22, 2015.

16. Yarden Y and Pines G: The ERBB network: At last, cancer therapy meets systems biology. Nat Rev Cancer 12: 553-563, 2012.

17. Hynes NE and Lane HA: ERBB receptors and cance: The complexity of targeted inhibitors. Nat Rev Cancer 5: 341-354, 2005.

18. Wang H, Xi Q and Wu G: Fatty acid synthase regulates invasion and metastasis of colorectal cancer via Wnt signaling pathway. Cancer Med 5: 1599-1606, 2016.

19. Li N, Bu X, Tian X, Wu P, Yang L and Huang P: Fatty acid synthase regulates proliferation and migration of colorectal cancer cells Via HER2-PI3K/Akt signaling pathway. Nutr Cancer 64: 864-870, 2012. 
20. Nikolayeva $\mathrm{O}$ and Robinson MD: edgeR for Differential RNA-seq and ChIP-seq Analysis: An application to stem cell biology. Methods Mol Biol 1150: 45-79, 2014.

21. Subramanian A, Tamayo P, Mootha VK, Mukherjee S, Ebert BL, Gillette MA, Paulovich A,Pomeroy SL, Golub TR, Lander ES and Mesirov JP: Gene set enrichment analysis: A knowledge-based approach for interpreting genome-wide expression profiles. Proc Natl Acad Sci USA 102: 15545-15550, 2005

22. Gazzoni GF, Fraga MB, Ferrari ADL, Soliz PDC, Borges AP, Bartholomay E, Kalil CAA, Giaretta V and Rohde LEP Predictors of total mortality and echocardiographic response for cardiac resynchronization therapy: A cohort study. Arq Bras Cardiol 109: 569-578, 2017 (In English, Portuguese).

23. Kang JS, Lee S, Son D, Han Y, Lee KB, Kim JR, Kwon W, Kim SW and Jang JY: Prognostic predictability of the new American Joint Committee on Cancer 8th staging system for distal bile duct cancer: Limited usefulness compared with the 7th staging system. J Hepatobiliary Pancreat Sci 25: 124-130, 2018.

24. Liu L, Shi M, Wang Z, Lu H, Li C, Tao Y, Chen X and Zhao J: A molecular and staging model predicts survival in patients with resected non-small cell lung cancer. BMC Cancer 18: 966, 2018.

25. Ohtsuki I and Morimoto S: Troponin: Regulatory function and disorders. Biochem Biophys Res Commun 369: 62-73, 2008.

26. Wei B and Jin JP: TNNT1, TNNT2, and TNNT3: Isoform genes, regulation, and structure-function relationships. Gene 582: 1-13,2016

27. Van Spaendonck-Zwarts KY, Van Rijsingen IA, Van den Berg MP, Lekanne Deprez RH, Post JG, van Mil AM, Asselbergs FW, Christiaans I, van Langen IM, Wilde AA, et al: Genetic analysis in 418 index patients with idiopathic dilated cardiomyopathy: Overview of 10 years' experience. Eur J Heart Fail 15: 628-636, 2013

28. Kong SW, Hu YW, Ho JW, Ikeda S, Polster S, John R, Hall JL, Bisping E, Pieske B, dos Remedios CG and Pu WT: Heart failure-associated changes in RNA splicing of sarcomere genes. Circ Cardiovasc Genet 3: 138-146, 2010.

29. Kozlovski I, Siegfried Z, Amar-Schwartz A and Karni R: The role of RNA alternative splicing in regulating cancer metabolism. Human Genet 136: 1113-1127, 2017.

30. Munkley J, Livermore K, Rajan P and Elliott DJ: RNA splicing and splicing regulator changes in prostate cancer pathology. Hum Genet 136: 1143-1154, 2017.
31. Lokody I: Alternative splicing: Aberrant splicing promotes colon tumour growth. Nat Rev Cancer 14: 382-383, 2014.

32. Zong Z, Li H, Yi C, Ying H, Zhu Z and Wang H: Genome-wide profiling of prognostic alternative splicing signature in colorectal cancer. Front Oncol 8: 537, 2018.

33. Adams JE III, Bodor GS, Dávila-Román VG, Delmez JA, Apple FS, Ladenson JH and Jaffe AS: Cardiac troponin I. A marker with high specificity for cardiac injury. Circulation 88 : 101-106, 1993.

34. De Luca A, Carotenuto A, Rachiglio A, Gallo M, Maiello MR, Aldinucci D, Pinto A and Normanno N: The role of the EGFR signaling in tumor microenvironment. J Cell Physiol 214: 559-567, 2008

35. Mallini P, Lennard T, Kirby J and Meeson A: Epithelial-tomesenchymal transition: What is the impact on breast cancer stem cells and drug resistance. Cancer Treat Rev 40: 341-348, 2014.

36. Spano JP, Fagard R, Soria JC, Rixe O, Khayat D and Milano G Epidermal growth factor receptor signaling in colorectal cancer: Preclinical data and therapeutic perspectives. Ann Oncol 16: 189-194, 2005.

37. Hung CY, Yeh TS, Tsai CK, Wu RC, Lai YC, Chiang MH, Lu KY, Lin CN, Cheng ML and Lin G: Glycerophospholipids pathways and chromosomal instability in gastric cancer: Global lipidomics analysis. World J Gastrointest Oncol 11: 181-194, 2019.

38. Mika A, Kobiela J, Czumaj A, Chmielewski M, Stepnowski P and Sledzinski T: Hyper-elongation in colorectal cancer tissue-cerotic acid is a potential novel serum metabolic marker of colorectal malignancies. Cell Physiol Biochem 41: 722-730, 2017.

39. Mirnezami R, Spagou K, Vorkas PA, Lewis MR, Kinross J, Want E, Shion H, Goldin RD, Darzi A, Takats Z, et al: Chemical mapping of the colorectal cancer microenvironment via MALDI imaging mass spectrometry (MALDI-MSI) reveals novel cancer-associated field effects. Mol Oncol 8: 39-49, 2014.

This work is licensed under a Creative Commons

Attribution-NonCommercial-NoDerivatives 4.0 International (CC BY-NC-ND 4.0) License. 